dius, der denselben Abschnitt aus dem Werk des Maximus aus-

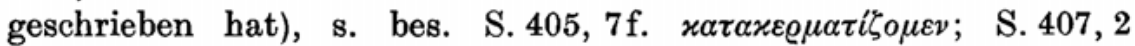

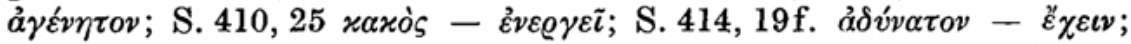
S. 415, 7f. $a \lambda \lambda \eta^{\prime} \lambda o \iota \varsigma ~-~ a ̉ v \tau i x \varepsilon \iota \tau \alpha \iota$. Die Annahme, daß Eus. eine fehlerhafte Hs. des Maximus benützt habe, verbietet uns die Tatsache, daß diese Fehler den Sinn stören (mit einem solchen Text würde sich Eus. schwerlich abgefunden haben). Wir müssen demnach annehmen, daß sich diese Fehler nach der Abfassung der P. E., aber noch vor 360 in den Archetypus unserer Hss. eingeschlichen haben.

\title{
Die Ausgaben
}

Erst 74 Jahre nach dem Erscheinen der lateinischen Übersetzung des Georgios von Trapezunt (s. S. XXVIII) erschien die erste Ausgabe des Originaltextes: Eusebii PamphiliEvangelicae Praeparationis lib. XV. Lutetiae. Ex officina Rob. Stephani 1544. Fol. Robert Estienne (Etienne), Vater des berühmteren Henry (Henricus Stephanus), hat durch die Beseitigung der gröbsten Versehen die Grundlage des Vulgattextes der P. E. geschaffen. Die Nummern seiner (einer Unterteilung entbehrenden) Seiten stehen auf den Rändern der folgenden Ausgaben (außer der von Heinichen, der von Gifford und meiner). $\mathrm{DaB}$ er Hss. der kgl. Bibliothek von Paris benützt hat, bezeugt er S. 1: EX BIBLIOTHECA REGIA. Welche Hss. es waren, wird im Zusammenhang mit der Besprechung der folgenden Ausgabe erörtert werden.

EusebiiPamphiliCaesareae Palaestinae episcopi Praeparatio Evangelica. Franciscus Vigerus Rothomagensis, Societ. Jesu Presbyter, ex MMSS. CODD. et laudatis ab ipsomet Eusebio Scriptoribus recensuit, Latine vertit $^{1}$ ), Notis illustravit. Parisiis $\left.1628^{2}\right)$. Fol. Durch diese Ausgabe erhielt der Vulgattext seine endgültige Form. Über die von Vigerus und seinem Vorgänger benutzten Hss. äußert er sich in der Praefatio S. III (wieder abgedruckt bei Gaisford IV 145): Novam ergo a capite interpretationem aggressi, duos ex Bibliotheca Regia... Manuscriptos Codices accepimus, quorum altero Stephanus in Graeca editione usus videtur, alterum ei saepe dissimillimum reperi. Accesserunt variae lectiones ex Richardi Montacutii libro descriptae, quarum mihi copiam aliquanto serius fecit $D$. Morellus . . ; usu cum in aliis non contemnendo, tum in lacunis quibusdam explendis fere praecipuo." Da sich keine Spur einer Benutzung der Pariser Hss. A und B (von denen

1) Diese Übersetzung war ganz neu, keine Neuauflage der des Georgios von Tr., s. Vigerus' Praef. an der gleich zu zitierenden Stelle.

2) Ein Nachdruck erschien 1688 in Leipzig (fälschlich Köln als Druckort angegeben). 
letztere sich damals noch gar nicht in der kgl. Pariser Bibliothek befand, A in Fontainebleau) durch Stephanus oder Vigerus zeigt, bleiben die Kodizes C, D und E. Da ferner Vigerus Lesarten des Codex R. Montacutii ( = C, s. S. XLI) aliquanto serius erhalten zu haben versichert, bleiben als Grundlage des Vulgattextes D und E. In der Tat liegen dem Text sowohl des Stephanus wie des Vigerus diese beiden Hss. zugrunde. Dafür ein paar Belege:

1. für DE: Bd.I S. 180, $\left.12 \lambda_{\iota} \mu \nu \alpha ́ \zeta \varepsilon เ v\right]$ 'Alter ex manuscr. $\lambda_{\iota} \mu \nu \alpha ́-$

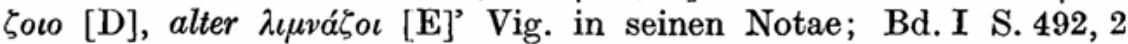

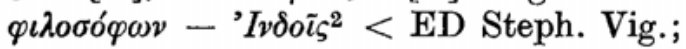

2. für E: Bd.I S. 112, $2 \pi \lambda \varepsilon i \sigma \tau \eta \nu$ E Steph., $\pi \lambda \varepsilon i \sigma \tau \alpha$ (falsch)

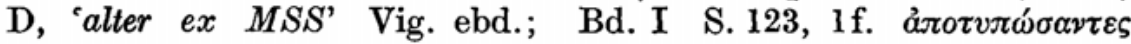

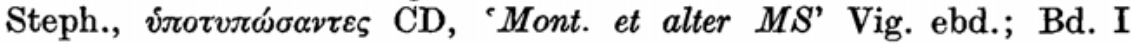

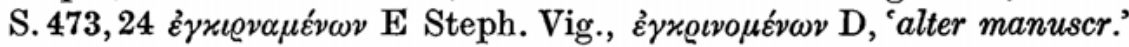

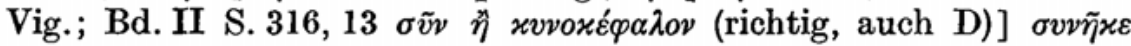

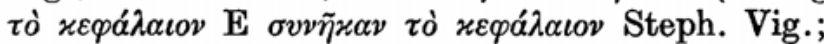

3. für D: Bd. I S. 36, $8 \tau \tilde{\eta}_{\varsigma^{2}}$ (richtig)] xai nur D, daraus Steph. und von ihm an alle bisherigen Herausgeber (auch Gifford); Bd. I S. 132, 4 xai à éoos (richtig, auch D)] < E Steph., 'additum est ex

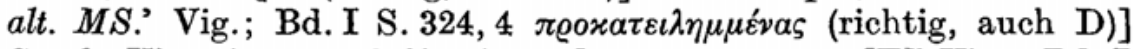

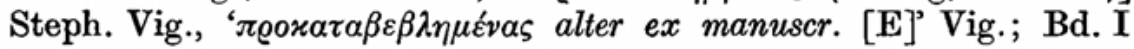

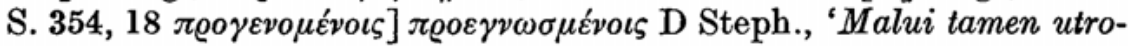

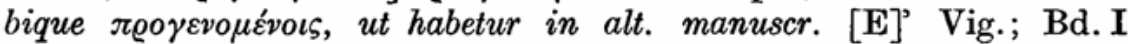

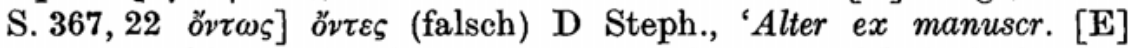

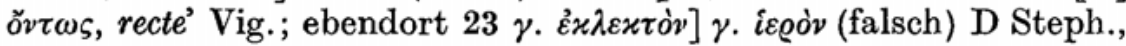

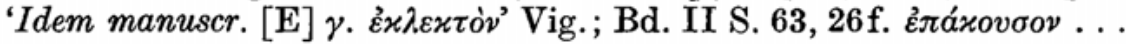

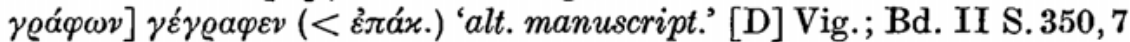

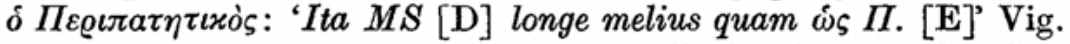

Vigerus (eigentlich Viguier), ein trefflicher Kenner des Griechischen ${ }^{1}$ ), hat sich um Eusebius noch größere Verdienste als Stephanus erworben, nicht bloß durch Verbesserungen, sondern auch durch Erklärungen (in den beigegebenen Notae), durch seine im ganzen vortreffliche Übersetzung (wieder abgedruckt bei Gaisford) und durch den Index rerum et nominum, den mit geringfügigen Änderungen noch Gifford übernommen hat. Die Nummern seiner durch die Buchstaben (a) bis d unterteilten Seiten haben alle folgenden Herausgeber außer Heinichen auf den Rändern beigefügt ${ }^{2}$ ).

1) Verfasser der (von G. Hermann neu herausgegebenen) Schrift De praecipuis graecae linguae idiotismis.

2) Einen (nicht sehr sorgfältig gemachten) Neudruck des Textes von Vigerus veranstaltete Migne, Patrologia Graeca Tom. XXI(Paris 1857), 21-1408. Beigegeben sind Sp. 1457-1666 Variae lectiones et notae des Seguierus, marchio a Sancto-Brissone (Séguier, marquis de Saint-Brisson). 
Eusebii Pamphili Praeparationis Evangelicae libri XV. Multo emendatiores cum variis codic. Voss. lectionibus nunc primum divulgatis et selectis Vigeri suisque annotationibus edidit, indices adiecit Fridericus Adolphus Heinichen. Tom I. (Lib. I-VIII) Lipsiae 1842, Tom.II. (Lib. IX $-X V$ et indices) 1843. $4^{\circ}$. Unterhalb des Textes stehen Anmerkungen (Stellenangaben, Verbesserungen [hauptsächlich die des Vigerus, nur ganz wenige von Heinichen], Erklärungen). Trotz der Anpreisung "multo emendatiores" zeigt der Text dieser auf Grund der Ausgaben von Stephanus und von Vigerus gemachten, auf sehr schlechtem Papier gedruckten und vom Verfasser eigenwillig und unübersichtlich eingeteilten Ausgabe ${ }^{1}$ ) keinen Fortschritt.

Die erste moderne Ausgabe ist folgende:

Eusebii Pamphili Evangelicae Praeparationis libri XV. Ad codices manuscriptos recensuit Thomas Gaisford, 4 Bde., Oxonii (e typographeo Academico) $18434^{\circ}$.

Auf jeder Seite dieser auf sehr schönem Papier gedruckten Ausgabe stehen der Text, der kritische Apparat und Vigerus' lateinische Übersetzung; der vierte Bd. umfaßt außer dem 15. Buch Vigerus' Dedicatio, seine Praefatio, seine Notae, eine Diatribe De Aristobulo von L. C. Valckenaer nebst Petri Wesselingii . . . De fragmento Orphei und De Aristobulo Iudaeo sowie die Indices.

Gaisford, der die Hss. A B C D E F G H I für sich hat vergleichen lassen, bietet einen ausführlichen kritischen Apparat, über dem freilich die Vulgata des Steph. und Vig. in meist vornehmer Unberührtheit thront, so daß H. Diels' scharfes Urteil (Doxographi Graeci2 $^{2}$, Berol. 1929, 43, vgl. auch 159) nicht unberechtigt ist ${ }^{2}$ ). Auf testimonia ex silentio ist bei ihm überhaupt kein Verlaß; dadurch sind Duebner (in der Ausgabe des Ezechiel) und Niese (Josephus, Gegen Apion) oft arg irregeführt worden. Von Gaisford rührt die Einteilung der Kapitel in Paragraphen her.

Eusebii Caesariensis opera. Recognovit Guil. Dindorfius, Vol. I. (Praeparationis evangelicae libri $I-X$ ) und Vol: II. (Libri $X I-X V$ nebst Indices). Lipsiae (Teubner) 1867.

In der Praefatio (I S. III-XXV) werden zum erstenmal die Hss. (bes. A und $\mathrm{H}$ ) ausführlicher besprochen, desgleichen die lateinische Übersetzung des Georgios. Eigene Kollationen scheint Dindorf nicht gemacht zu haben, sondern auf Gaisfords handschriftlichem Material zu fußen. Zur Herstellung des Textes hat dieser aus-

1) Die Seitennummern der Ausgaben sowohl des Steph. als auch des Vig. fehlen gänzlich.

$\left.{ }^{2}\right)$... ex Gaisfordii indigesta mole ... quanta perversitate Eusebii illa editio conflata sit, nolo conqueri. 
gezeichnete Kenner des Griechischen und durch die Herausgabe vieler Autoren wohlgeübte Gelehrte manches beigetragen. Ein kritischer Apparat fehlt leider, und die in der Praef. S. IV und XXIVf. angekündigten Annotationes sind nie erschienen.

Eusebii Pamphili Evangelicae Praeparationis libri XV. Ad codices manuscriptos denuo collatos recensuit, Anglice nunc primum reddidit, notis et indicibus instruxit $\boldsymbol{E}$. H. Gifford, Oxonii (e typographeo Academico) 1903. Tom. I (Buch I-IX), II (Buch X-XV nebst Indices und Appendix mit Scholia quaedam potiora); Tom. III (zweiteilig) enthält die englische Übersetzung ${ }^{1}$ ), Tom. IV Notae in englischer Sprache. $4^{\circ}$.

Diese sehr schön ausgestattete Ausgabe bedeutet einen gewaltigen Fortschritt gegenüber allen früheren Ausgaben. Ganz neue Kollationen (darunter die des zum erstenmal verwerteten Kodex 0 ) haben die Grundlage für den Text gebildet, der kritische Apparat ist reichhaltig und steht in organischer Verbindung mit dem Text (ist also nicht eine bloße Zugabe wie bei Gaisford), die Übersetzung bietet dem Leser trotz mancher Mißverständnisse eine gute Beihilfe, und in den Notes stehen vortreffliche Hinweise und Erläuterungen. Leider hat sich aber Gifford vom Vulgattext nicht völlig frei gemacht, ferner $A$ überschätzt und $N$ verschmäht. Im übrigen verweise ich auf die ausführliche Besprechung dieser Ausgabe durch O. Stählin, Wochenschrift f. klass. Philol. 22 (1905) 1007-1011 und 1027-1031 sowie auf die von J. Bidez, Revue critique N. S. 61 (1906) 506-509.

\section{Inhalt der P. E.}

Eusebius gibt selber im ersten Kapitel des XV. Buches eine ausgezeichnete Rekapitulation des Inhaltes (unter Zusammenfassung der Bücher zu Triaden).

\section{Zeit der Abfassung der P. E.}

Einen festen Anhalt bietet die Anspielung auf den Betrug des heidnischen Propheten Theoteknos (Ende 313 oder Anfang 314) Bd. I S. 168, 1 Iff. (s. meine dortige Bemerkung). Diese Stelle muß also um diese Zeit (offenbar unter dem frischen Eindruck dieses Ereignisses) geschrieben sein. Da anderseits zur Zeit der Abfassung

1) Außer der lateinischen Übersetzung des Georgios und der des Vigerus sowie der englischen Übersetzung Giffords gibt es noch eine französische von Migne (in dem Sammelwerk Démonstrations Évangéliques, Paris 1842-1853) und eine von Séguier de Saint-Brisson (Paris 1846). 